\title{
QoS based fair load-balancing: paradigm to IANRA routing algorithm for wireless networks (WNs)
}

\begin{abstract}
In this paper, a new algorithm, Intelligent agent AntNet based Routing Algorithm (IANRA) is proposed to enhance load balancing strategy in Wireless Networks (WNs). IANRA is based on Ants behaviour with some important factors such as: the specific self-organizing behaviour of ant colonies, the shortest path discovery and the related framework of Ant Colony Optimization (ACO). The main focus in IANRA is to find optimum and near optimum route by means of Genetic Algorithm (GA) using breeding capability of ants. Here, ants can produce a number of generations with the target to discover an optimized route. Hence IANRA is able to prevent of the difficulties which exist in existing routing algorithms, such as Ad hoc On-Demand Distance Vector (AODV), AntNet etc. The obtained results show that the efficiency of IANRA algorithm is better than AODV and AntNet or any other related algorithm. In addition, the proposed algorithm (IANRA) is able to reduce the end-toend delay and increase the packet delivery ratio significantly.
\end{abstract}

Keyword: Ant colony optimization (ACO); IANRA; Load balancing; QoS; Routing algorithm; Wireless communication networks 